\title{
Computation of time-lapse differences with 3D directional frames
}

\author{
Moritz Beyreuther ${ }^{1}$, Jamin Cristall ${ }^{\dagger \dagger}$, and Felix J. Herrmann ${ }^{2}$ \\ ${ }^{1}$ Department of Earth and Environmental Sciences, Ludwig Maximilian Universitaet, Munich, Germany \\ ${ }^{2}$ Department of Earth and Ocean Sciences, University of British Columbia, Vancouver, Canada \\ ${ }^{\dagger}$ now with Cameco Corporation, Saskatoon, Canada
}

\begin{abstract}
Summary
We present an alternative method of extracting production related differences from time-lapse seismic data sets. Our method is not based on the actual subtraction of the two data sets, risking the enhancement of noise and introduction of artifacts due to local phase rotation and slightly misaligned events. Rather, it mutes events of the monitor survey with respect to the baseline survey based on the magnitudes of coefficients in a sparse and local atomic decomposition. Our technique is demonstrated to be an effective tool for enhancing the time-lapse signal from surveys which have been cross-equalized.
\end{abstract}

\section{Introduction}

A major challenge in time-lapse seismic is that the differences caused by small changes in acquisition geometry and processing often overshadow the differences caused by true changes to the reservoir [1]. Cross-equalization, as discussed by [12], has been widely successful in correcting for many of the distortions that inevitably arise when two 3D surveys are shot in different calendar years and with variable source and receiver positions. This technique involves re-binning the two data sets to a common grid, applying a space and time variant amplitude envelope balance, running a first pass of matched filter corrections for global amplitude, bandwidth, phase, and static shift corrections, followed by a dynamic warp to align mispositioned events; and, finally, running a second pass of constrained space-variant matched filter operators [13].

A difficulty in cross-equalization is to design a set of matched filters that will correct for acquisition and processing related differences without degrading the true time-lapse signal. Using an overly conservative approach will assure that time-lapse effects are preserved but will fail to correct for all of the differences related to acquisition and processing. When two seismic volumes are subtracted in the presence of this residual colored noise, as well as random white noise, non-production related differences will be enhanced and true reservoir changes may be difficult to interpret.

A variety of techniques have been proposed to more effectively difference 3D seismic data volumes in the presence of white noise and systematic differences related to acquisition and processing. [9] calculated the robust difference, which is effectively smoothing prior to differencing. [11] achieved a measurable improvement over differencing in the time domain by separating out the time-lapse signal through singular value decomposition.
The technique that we propose involves decomposing the baseline and monitor surveys with atoms that are optimized for representing piecewise smooth images, such as seismic data [8]. The three-dimensional atoms we employ, called curvelets, are able to represent seismic data very efficiently because of their multiscale and multi-directional properties. This ability to represent the data with only a few coefficients allows us to decorrelate the time-lapse signal through a simple soft thresholding procedure. Our technique is robust with respect to local phase rotations, small reflector misalignments, and low SNR in both the baseline and monitor surveys.

In this paper, we reformulate the problem of computing timelapse difference cubes into the framework of solving a generic minimization problem. We describe the properties of the curvelet frame and explain how the minimization problem is solved by thresholding in the curvelet domain. The technique is illustrated with $2 \mathrm{D}$ synthetic examples and $3 \mathrm{D}$ results from the Ula field in the North Sea.

\section{The minimization problem}

We propose that computing time-lapse difference cubes can be recast into the framework of solving a generic minimization problem:

$$
\mathbf{b}=\mathbf{a}+\mathbf{d}+\mathbf{n}
$$

where $\mathbf{b}$ is the monitor survey, $\mathbf{a}$ is the baseline survey, $\mathbf{d}$ is the time-lapse difference, and $\mathbf{n}$ is white noise. An estimate of the time lapse difference is obtained by the following minimization:

$$
\hat{\boldsymbol{\delta}}=\arg \min _{\boldsymbol{\delta}} \frac{1}{2}\|\mathbf{b}-\mathcal{F} \boldsymbol{\delta}\|_{2}^{2}+\mu\|\boldsymbol{\delta}\|_{1, \mathbf{w}}
$$

with $\|\boldsymbol{\delta}\|_{1, \mathbf{w}}=\sum_{i=1}^{N}\left|w_{i} \delta_{i}\right|$ the weighted $\ell^{1}$-norm over the $N$ vector elements with weights $\mathbf{w}=|\boldsymbol{\delta}|$ and where $\mathcal{F}^{*}$ represents an atomic decomposition, called the analysis operator. $\mathcal{F}$ is the synthesis operator. Vectors $\boldsymbol{\alpha}, \boldsymbol{\beta}$, and $\delta$ represent the coefficients of $\mathbf{a}, \mathbf{b}$, and $\mathbf{d}$ in the atomic expansion. The operator .* represents a component wise product between $\boldsymbol{\alpha}$ and $\boldsymbol{\beta}$. Note that the second term is minimized in an $\ell^{1}$ sense. The use of the $\ell^{1}$ norm encourages the atom coefficients to be as sparse as possible. The control parameter $\mu$ governs the amount of emphasis on minimization of the misfit between the time-lapse signal and monitor survey versus minimization of the correlation between time-lapse signal and baseline survey. Essentially, our goal is to recover an estimate of the time-lapse signal that is minimally correlated with the baseline survey and maximally present in the monitor survey.

The question now is: how can we solve this minimization problem effectively? The work of [6], [10] and others has shown 


\section{Computation of time-lapse differences with 3D directional frames}

that, for a certain class of models, the above minimization problem can be solved by projecting the data onto an atomic representation that is optimal for that particular class of models. In doing so, most of the model's energy will reside in only a few coefficients. This allows the definition of a shrinkage operator that approximately solves the above minimization problem by soft thresholding the coefficients with

$$
S_{w}(x) \equiv \begin{cases}x-\operatorname{sign}(x) w & |x| \geq w \\ 0 & |x|<w\end{cases}
$$

$\mathcal{S}_{w}$ is a element-wise soft thresholding operator that shrinks the coefficients towards zero that are below the threshold $\mathbf{w}=$ $\mu|\boldsymbol{\alpha}|$. A second term containing the variance of several (e.g. 4) migrated Gaussian noise realizations may be added to the threshold within the domain defined by the atomic decomposition. This additional thresholding term improves the result by de-noising in conjunction with recovering the time-lapse signal.

The soft thresholding of Eq. 3 represents the first iteration of the following iterative scheme, which is based on the Landweber iterative method [4],[15]:

$$
\hat{\boldsymbol{\delta}}^{k}=\mathcal{S}_{\mathbf{W}}\left(\boldsymbol{\delta}^{k-1}+\mathcal{F}^{*}\left(\mathbf{b}-\mathcal{F} \boldsymbol{\delta}^{k-1}\right)\right) ; \quad k \in \mathbb{N}^{+}
$$

The symbol $k$ represents the iteration number. Eq 4 converges to a sparse set of coefficients, which form the solution to Eq. 2

\section{Curvelet atom decomposition}

The solution outlined above requires that the atomic decomposition is able to effectively represent the model with only a few coefficients. Curvelets, as described by [5],[3] constitute a relatively new family of non-separable wavelet bases that are designed to efficiently represent images displaying curve punctuated smoothness. This property makes curvelets suitable for representing reflectors, which may be faulted, within 3D seismic data volumes. Curvelets gain their success from their ability to align with the contours of a 3D or 2D object and represent it with only a few coefficients. In comparison, the wavelet transform requires many more coefficients to effectively represent the contours of a piecewise smooth object [14],[7]. Curvelets are not
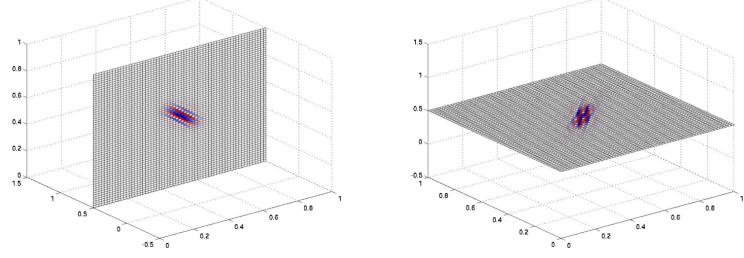

Fig. 1: A pair of slices through a 3D curvelet

an orthogonal basis but rather a tight frame. A frame is an overcomplete (linearly dependent) version of a basis. If the norm of the transformed signal remains linearly proportional to the norm of the original signal the frame is said to be a tight frame. Frames represent an image with redundant basis vectors, allowing more opportunity for the atoms to align with the contours of the image. This allows frames to represent an image with less coefficients than would be possible with a basis [5].

Both 3D and 2D curvelet atoms are anisotropically shaped and distinguished by their scale, angle, and position [1]. Each scale is represented at a number of angles, which double at every other scale. At each scale, angle, and position the curvelet atom is correlated (dot product) with the image to calculate the curvelet coefficient for that particular scale, angle, and position. The curvelet coefficient will be large if the atom aligns with the reflector and small if it does not. Piecewise continuous elements of an image, such as faulted reflectors, remain efficiently represented in the curvelet domain.

Since curvelets have the ability to represent seismic data very efficiently, they can be used to solve the minimization problem of Eq. 2 using the algorithm of Eq. 4. In this case, $\mathcal{F}^{*}$ represents the forward curvelet transform and $\mathcal{F}$ represents the inverse curvelet transform.

\section{D synthetic data examples}

An advantage of using curvelets in the formulation of Eq. 4 is that soft thresholding a sparse representation of a signal is robust under local phase rotation. This implies that the operation will also be robust with respect to small time shifts. These characteristics are illustrated by Fig. 2
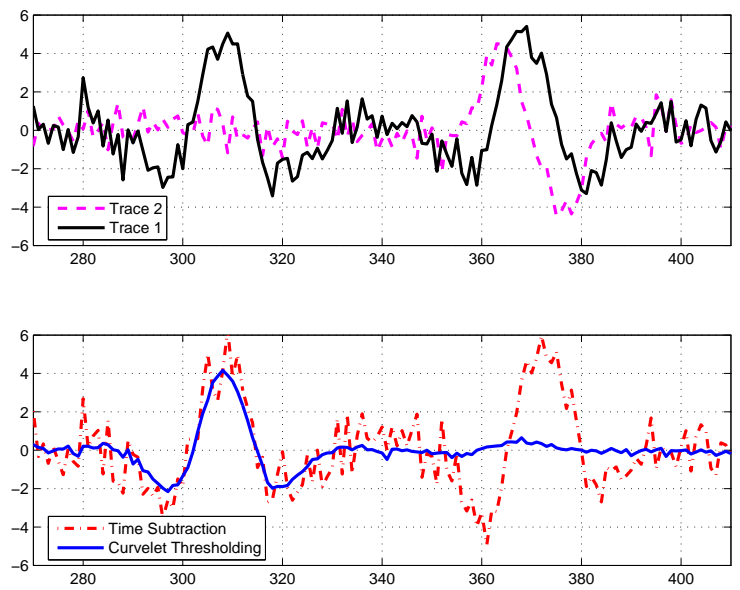

Fig. 2: Top: Two traces extracted from two hypothetical 2D seismic surveys. The reflector of Trace 2 was produced by taking the second reflector of Trace 1, time shifting it by two samples, and phase rotating it by $90^{\circ}$. Bottom: The corresponding two traces showing the results of subtracting the two 2D surveys in the time domain versus soft thresholding in the curvelet domain

Fig. 2(Top) shows two traces extracted from two hypothetical 2D seismic surveys. Different Gaussian noise realizations were added to each synthetic $2 \mathrm{D}$ section to produce noisy sections with a SNR of $0 \mathrm{~dB}$. Trace 1 exhibits two reflectors while Trace 2 shows only one reflector. The reflector of Trace 2 was produced by taking the second reflector of Trace 1 , time shifting it by two samples, and phase rotating it by $90^{\circ}$. Fig. 2 (Bottom) shows the corresponding two traces extracted from two $2 \mathrm{D}$ difference sections computed by subtraction in the time domain versus soft 


\section{Computation of time-lapse differences with 3D directional frames}

thresholding in the curvelet domain. It is evident that subtraction in the time domain results in a large amplitude difference at the second horizon. However, difference at the second horizon is small when curvelet domain soft thresholding is employed. This example illustrates that curvelet domain soft thresholding is robust with respect to local phase rotations as well as small reflector time shifts.

For our second synthetic example, we tested our methodology in the complex structural setting defined by the Marmousi velocity model [Fig. 3 (Top)]. This velocity model was constructed in 1988 at the Institut Français du Pétrole (IFP). It is based on geology from Angola, West Africa.

The P-wave velocity of the oil column in the lower anticlinal structure was increased from 3230 to $3600 \mathrm{~m} / \mathrm{s}$ in order to simulate depletion of the reservoir and inflow of water. We calculated the reflectivity of both the pre- and post-production velocity models, applied the scattering operator, and then added different Gaussian noise realizations to each of the scattered data sets. This produced both a pre-production and post-production synthetic $2 \mathrm{D}$ section with a SNR of $-4 \mathrm{~dB}$. Next, we migrated each synthetic section using a constant velocity migration with a different migration velocity. The pre-production synthetic was migrated with a constant velocity of $3480 \mathrm{~m} / \mathrm{s}$ and the postproduction synthetic was migrated with a constant velocity of $3500 \mathrm{~m} / \mathrm{s}$. This was done in order to simulate the processing differences and small reflector misalignments that might be present between two different survey vintages. Note that Gaussian noise will become colored noise upon migration.

Fig. 3 (Middle) shows the results of differencing the pre- and post-production synthetics in the time domain. It is evident that large amounts of difference arise where no production related velocity changes have occurred. This is mainly a result of reflector misalignments caused by using two different constant migration velocities. Fig. 3 (Bottom) shows the results of applying our curvelet domain soft thresholding scheme. It is evident that our methodology is able to detect the true reservoir velocity change in spite of the significant reflector misalignments and added colored noise. This result demonstrates that our methodology provides an interesting alternative to matched filtering schemes because it remains robust when the SNR is low.

\section{D real data example}

Two 3D seismic surveys were acquired at the Ula North Sea oil field in 1999 and 2002 respectively. The monitor survey was designed to be as similar to the baseline survey as possible and the two surveys were processed in parallel. In spite of this, repeatability was quite low $(N R M S \sim 150 \%)$ and upon subtraction it became evident that acquisition/processing related artifacts complicated the interpretation of true time-lapse effects.

We tested our methodology by extracting a sub-cube, from both the baseline and monitor surveys, around an area containing two injection wells; both of which injected a significant amount of gas between 1999 and 2002 and were converted to water and gas in 2001/2002. Our goal was to enhance the time lapse signal using the iterative curvelet domain soft thresholding scheme of Eq. 4 We proceeded by computing the 3D curvelet decompo-
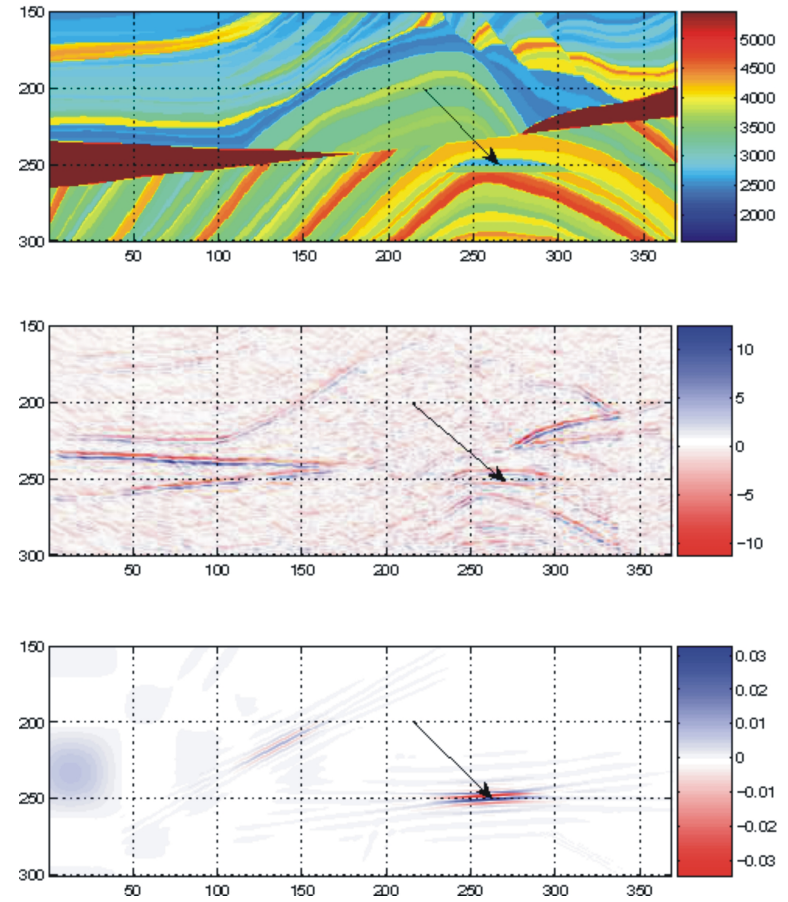

Fig. 3: Top: Pre-production Marmousi velocity model. The arrow indicates the position of the reservoir. Middle: Results of differencing the pre- and post- production synthetics in the time-domain Bottom: Results of extracting the time-lapse difference by soft thresholding in the curvelet domain. The color bar is indicative of the amplitude difference. The difference amplitudes are shrunk by the $\ell^{1}$ norm due to the low SNR in this example.

sition of both the baseline and monitor survey sub-cubes. This computation was performed in $3 \mathrm{D}$ all at once on the entire subcube. The time-lapse difference was calculated according to Eq. 4 with threshold $\boldsymbol{\alpha}$ given by the 1999 data set, $\boldsymbol{\beta}$ given by the 2002 data set, and $\mu=1$. In this case, it was not possible to calculate the additional denoising term of the threshold because the migration was done prior to our receiving the data and the migration operator was not known.

The results are illustrated by Fig. 4 Fig. 4 (Top) shows the time-lapse difference calculated by traditional subtraction projected onto the Top Ula reservoir horizon. Fig. 4(Bottom) shows the time-lapse difference calculated by our curvelet domain soft thresholding methodology projected onto the Top Ula horizon. It is believed that acquisition/processing related artifacts have been reduced and the time-lapse signal has been enhanced.

\section{Discussion}

We presented an alternative method of computing time-lapse seismic difference cubes. This method is based upon decomposing the data in a sparse and local frame. The 3D curvelet atoms we employ are able to represent seismic data very efficiently because of their multi-scale and multi-directional properties. This ability to represent the data with only a few coefficients allows us to decorrelate the time-lapse difference through a 


\section{Computation of time-lapse differences with 3D directional frames}

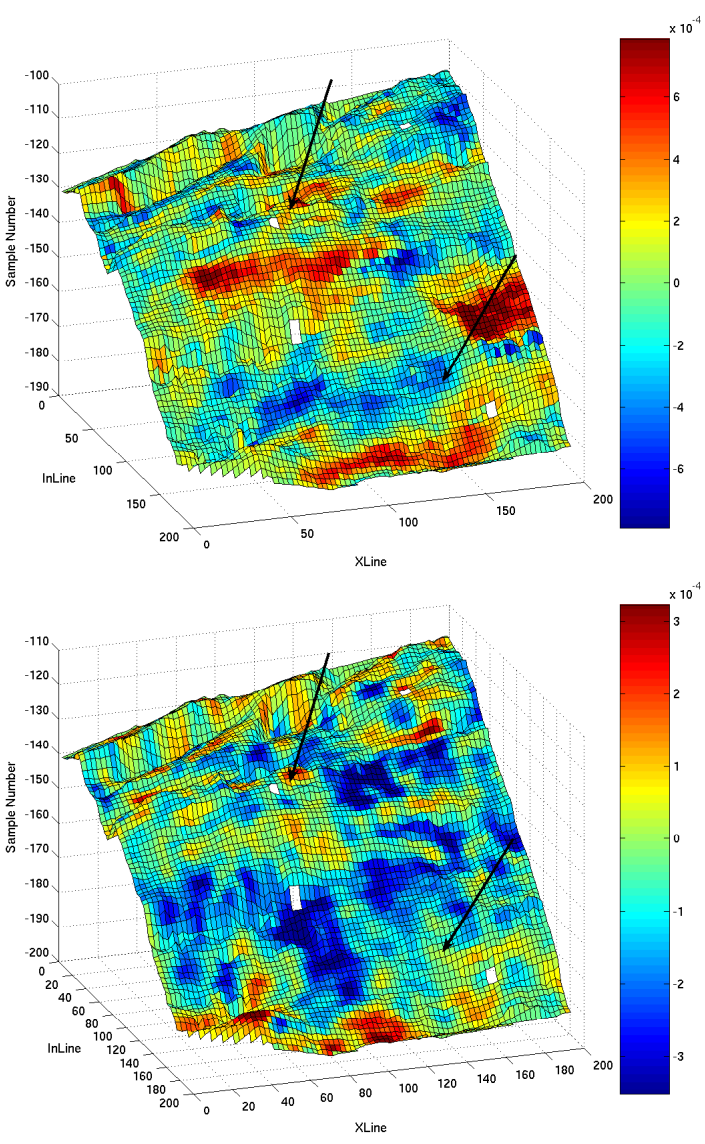

Fig. 4: Top: Time-lapse difference calculated by time domain subtraction projected onto the Top Ula reservoir horizon Bottom: Timelapse difference calculated by curvelet domain soft thresholding projected onto the Top Ula horizon. The color bar is indicative of the amplitude difference. Positive numbers correspond to brightening and negative numbers correspond to dimming. The two arrows indicate the positions of two injector wells.

simple soft thresholding procedure. Our technique appears to be an attractive addition to cross-equalization because it is robust with respect to local phase rotations, small reflector misalignments, and low SNR data.

\section{Acknowledgments}

The authors would like to thank Paul R. Gutowski and Per Gunnar Folstad of BP for their advice and thank the Ula license partners, BP Norge, Svenska Petroleum, and Dong Norge, for their generous permission to use their data. We would also like to thank the members of the Seismic Laboratory for Imaging and Modeling (Dupuis, Moghaddam, Hennenfent, and Paradis) for their thoughtful insights and fruitful discussions. The authors of the Digital Curvelet Transform [2] are gratefully acknowledged for allowing us to be the first group to apply their 3D curvelet code to seismic data. This work was in carried out as part of the SINBAD project with financial support secured, through ITF (the Industry Technology Facilitator), from the following corporations: BG Group, BP, ExxonMobil and Shell.
Additional funding was provided by NSERC Discovery Grant 22R81254.

\section{References}

[1] R. Calvert. 4d technology: where are we, and where are we going? Geophysical Prospecing, 53(2):161-171, 2005.

[2] E. Candes, D. Donoho, L. Demanet, and L. Ying. Curvelab: Fast discrete curvelet transform. URL www. curvelets.org

[3] E. J. Candès and F. Guo. New multiscale transforms, minimum total variation synthesis: Applications to edgepreserving image reconstruction. Signal Processing, pages 1519-1543, 2002. URL http://www.acm.caltech.edu/ ^emmanuel/publications.html

[4] I. Daubechies, M. Defrise, and C. de Mol. An iterative thresholding algorithm for linear inverse problems with a sparsity constrains. CPAM, (11):1413-1457, 2005.

[5] M. Do and M. Vetterli. Beyond wavelets, chapter Contourlets. Academic Press, 2002

[6] D. L. Donoho and I. M. Johnstone. Minimax estimation via wavelet shrinkage. Annals of Statistics, 26(3):879-921, 1998. URL citeseer.nj.nec.com/donoho92minimax.html

[7] F. Herrmann and G. Hennenfent. Non-linear data continuation with redundant frames. In CSEG National Convention, 2005. URL http://zoozoo.eos.ubc.ca/ ₹felix/private/ Herrmann_F_Non_Linear_Data_Continuation 101S0201.doc Accepted.

[8] F.J. Herrmann and P.P. Moghaddam. Sparseness- and continuityconstrained seismic imaging with curvelet frames. 2005. URL http://zoozoo.eos.ubc/ ^felix/private/Migr. pdf Submitted for publication.

[9] M. Landrø, O. A. Solheim, E. Hilde, B. O. Ekren, and L. K. Strøen. The gullfaks 4d seismic study. Petroleum Geosience, 5(3):213$228,1999$.

[10] S. G. Mallat. A wavelet tour of signal processing. Academic Press, 1997.

[11] F. J. L. Reid, A. Bertrand, A. T. McInally, and C. M. Beth. 4d signal enhancement using singular-value decomosition: appliction to mapping oil-water contact movement across the nelson field. Geophysical Prospecing, 53(2):253-263, 2005.

[12] J. Rickett and D. E. Lumley. A cross-equalization processing flow for off-the-shelf 4-D seismic data. In 68th Ann. Internat. Mtg, pages 16-19. Soc. of Expl. Geophys., 1998.

[13] J. Rickett and D. E. Lumley. Cross-equalization data processing for time-lapse seismic reservoir monitoring: A case study from the Gulf of Mexico. Geophysics, 66(4):1015-1025, 2001.

[14] J. L. Starck, E. J. Candès, and D. L. Donoho. The curvelet transform for image denoising. IEEE Transactions on Imaged Processing, 11(6), 2002.

[15] J. L. Starck, M. Elad, and D. Donoho. Redundant multiscale transforms and their application to morphological component separation. Advances in Imaging and Electron Physics, 132, 2004. 